\title{
POWER SERIES WITH ALMOST PERIODIC COEFFICIENTS
}

\section{J. HENNIGER ${ }^{1}$}

In a recent note [1], Davenport has given rather general conditions for the convergence of the series

$$
f(x)=\sum_{n=-\infty}^{\infty} a_{n} e(n \gamma) /(1-x e(n \alpha))
$$

and for the existence of its alternative expression

$$
f(x)=\sum_{\nu=0}^{\infty} g(\nu \alpha+\gamma) x^{\nu} \quad \text { where } \quad g(t)=\sum a_{n} e(n t) .
$$

Here $|x|<1, \gamma$ is real, $\alpha$ irrational and $e(\theta)=e^{2 \pi i \theta}$. He proves under the same conditions that

$$
(1-r) f(r e(-k \alpha)) \rightarrow a_{k} e(k \gamma) \quad \text { as } r \rightarrow 1 \quad(0<r<1),
$$

and that the corresponding limit at $x=e(\theta)$, where $\theta$ is not congruent $(\bmod 1)$ to an integral multiple of $\alpha$, is zero.

P. Szüsz has pointed out that for the proof of (1) and (2) Davenport's conditions can be reduced to the following:

(a) $\lim _{N \rightarrow \infty} \sum_{|n| \leq N} a_{n} e(n t)$ exist for each real $t$, and

(b) $\left|\sum_{|n| \leqslant N} a_{n} e(n t)\right|<K<\infty$ uniformly in $N$ and $t$.

That is, the convergence of $\sum a_{n}$ and condition (6) in Davenport's paper can be replaced by (a).

The purpose of this note is to generalize the above results in a couple of directions. It is to be noticed that the power series coefficients in (2), $A_{\nu}=g(\nu \alpha+\gamma)$, constitute in some general sense an almost periodic sequence with the Fourier expansion

$$
A_{\nu}=g(\nu \alpha+\gamma)=\sum_{n} a_{n} e(n \gamma) e^{2 \pi i n \alpha \nu} .
$$

We shall show that the Fourier exponents in (4), $n \alpha$ (and correspondingly, the same sequence in (1)) can be replaced by an arbitrary set of numbers $\alpha_{n}$. Also, instead of convergence of the Fourier series, we shall only require that $A_{\nu}$ be the pointwise limit of a sequence of trigonometric polynomials. For the proof of (1) and (2) bounded con-

Received by the editors July 11, 1966.

1 This research was carried out during tenure of a National Research Council (Canada) Postdoctorate Fellowship. 
vergence of the approximating polynomials will be required; for the proof of (3) a more uniform type of convergence must be assumed.

Definition. We call $A_{\nu}, \nu=0,1,2, \cdots$, a boundedly almost periodic sequence if $A_{\nu}$ is the limit of a uniformly bounded sequence of trigonometrical polynomials:

$$
A_{\nu}=\lim _{N \rightarrow \infty} \sum_{n} a_{n}(N) e^{2 \pi i \alpha_{n} \nu} \quad \text { for each } \nu .
$$

The exponents $\alpha_{n}$ may be complex, though it is obviously necessary that $\operatorname{Im}\left(\alpha_{n}\right) \geqq 0$. We may assume without loss generality that $0 \leqq \operatorname{Re}\left(\alpha_{n}\right)<1$.

Such a sequence is $A_{\nu}=g(\nu \alpha)$ where $\alpha$ is irrational and $g(t)$ is a bounded complex-valued function of period 1, with discontinuities only at rational points. The Féjer polynomials (which in this case are uniformly bounded and converge at each point of continuity, hence at each $\nu \alpha$ ) can serve as the approximating polynomials (see [2, p. 89]). In particular $A_{\nu}=(\nu \alpha)$ where $(t)$ is the fractional part of $t$, satisfies the conditions of the definition.

TheOREM 1. Let $A_{\nu}$ be boundedly almost periodic with the approximating polynomials $A_{\nu}(N)=\sum a_{n}(N) e\left(\alpha_{n} \nu\right)$. Then the series

$$
f(x)=\lim _{N \rightarrow \infty} \sum_{n} a_{n}(N) /\left(1-x e\left(\alpha_{n}\right)\right)
$$

converges in $|x|<1$, and has there the power series

$$
f(x)=\sum_{\nu=0}^{\infty} A_{\nu} x^{\nu}
$$

Proof. From $\left[1-x e\left(\alpha_{n}\right)\right]^{-1}=\sum_{v=0}^{\infty} e\left(\nu \alpha_{n}\right) x^{\nu}$ we have

$$
\begin{aligned}
\sum_{n} a_{n}(N) /\left(1-x e\left(\alpha_{n}\right)\right) & =\sum_{\nu}\left[\sum_{n} a_{n}(N) e\left(\alpha_{n} \nu\right)\right] x^{\nu} \\
& =\sum_{\nu=0}^{\infty} A_{\nu}(N) x^{\nu} .
\end{aligned}
$$

Let $A$ be the upper bound of $\left|A_{\nu}(N)\right|$ over $N$ and $\nu$. Given any $\epsilon>0$, we choose $M$ so large that $2 A \sum_{M}^{\infty}|x|^{\nu}$ is less than $\epsilon / 2$. We then let $N_{\epsilon}$ be so large that for all $N>N_{\epsilon}$

$$
\sup _{0 \leqq \nu \leqq M}\left|A_{\nu}(N)-A_{\nu}\right|<(\epsilon / 2)(1-|x|) .
$$

Then for all $N>N_{\epsilon}$ we have 


$$
\begin{aligned}
\left|\sum_{\nu}\left(A_{\nu}(N)-A_{\nu}\right) x^{\nu}\right| & \leqq(\epsilon / 2)(1-|x|) \sum_{0}^{M}|x| \nu+2 A \sum_{M}^{\infty}|x|^{\nu} \\
& <\epsilon / 2+\epsilon / 2=\epsilon .
\end{aligned}
$$

The convergence of (7) to (6) as $N \rightarrow \infty$, and the equality of (6) and (5) immediately follow.

In order to prove the analogue of (3) we require the following concepts.

Definition. A set of nonnegative integers $D$ is said to have maximum density $\delta$ if $N_{D} / N<\delta$ for all $N$ greater than some $M_{\delta}$, where $N_{D}$ is the number of integers in $D$ less than $N$. We will say that $A_{\nu}(N) \rightarrow A_{\nu}$ quasi-uniformly if for each $\delta>0$ the convergence is uniform on a set $I-D$, where $D$ has maximum density $\delta$ ( $I$ is the set of nonnegative in tegers).

TheOREM 2. Let $A_{\nu}$ be boundedly almost periodic with the quasiuniformly convergent approximating polynomials

$$
A_{\nu}(N)=\sum_{n} a_{n}(N) e\left(\alpha_{n} \nu\right) .
$$

Suppose that $a_{n}(N) \rightarrow a_{n}$ as $N \rightarrow \infty$, for each $n$. If $f(x)$ is as defined in (5) and $\alpha_{k}$ is real, then

$$
(1-r) f\left(r e\left(-\alpha_{k}\right)\right) \rightarrow a_{k} \quad \text { as } \quad r \rightarrow 1 \quad(0<r<1) .
$$

Proof. For $N>k$ we put $f_{N}(x)=\sum_{n} a_{n}(N) /\left(1-x e\left(\alpha_{n}\right)\right)$, and $f(x)$ $=f_{N}(x)+R_{N}(x)$, so that (as in the proof of Theorem 1)

$$
R_{N}(x)=\sum_{\nu}\left[A_{\nu}-A_{\nu}(N)\right] x^{\nu} .
$$

We first show that $R_{N}(x)$ can be made arbitrarily small by making $N$ sufficiently large. Given any $\delta>0$, let $D$ be a set of natural numbers of maximum density $\delta$ (with $N_{D} / N<\delta$ for $N>M_{\delta}$ ) such that the convergence of $A_{\nu}(N)$ is uniform on $I-D$. We let $D^{\prime}$ be the set of those $\nu$ in $D$ with $|\nu| \geqq \max \left[M_{\delta}, 1 / \delta\right]$. Then given any $\epsilon>0$, let $N_{\epsilon}$ be such that $\left|A_{\nu}(N)-A_{\nu}\right|<\epsilon / 4$ for all $N>N_{\epsilon}$, and $\nu \notin D^{\prime}$. Then

$$
\begin{aligned}
\left|R_{N}(x)\right| & \leqq \sum_{\nu=0}^{\infty}\left|A_{\nu}(N)-A_{\nu}\right||x|^{\nu} \\
& <(\epsilon / 4) /(1-|x|)+2 A \sum_{\nu \in D^{\prime}}|x|^{\nu} .
\end{aligned}
$$

This last series will be largest in value when the $\nu$ take on the smallest possible values under the restrictions on $D^{\prime}$, and the set $D^{\prime}$ with small- 
est possible members is obviously $\{j / \delta: j=1,2, \cdots\}$. Hence the series is less than

$$
\sum_{j=1}^{\infty}|x|^{j / \delta}=1 /\left(|x|^{-1 / \hat{\delta}}-1\right) \sim \delta /(1-|x|) \quad \text { as }|x| \rightarrow 1,
$$

since $r^{p}-1 \sim p(r-1)$ as $r \rightarrow 1$. Since $\delta$ was arbitrarily chosen, it follows that we can find $N_{\epsilon}$ and $r(\epsilon)$ with the property that for $N>N_{\epsilon}$ and $r(\epsilon)<|x|<1$,

$$
(1-|x|)\left|R_{N}(x)\right|<\epsilon / 2 .
$$

Now choose $N>N_{\epsilon}$ so large that $\left|a_{k}(N)-a_{k}\right|<\epsilon / 4$. Since the expression for $f_{N}(x)$ contains only a finite number of terms, we can choose $r(\epsilon)$ so close to 1 that

$$
\left|(1-r) f_{N}\left(r e\left(-\alpha_{k}\right)\right)-a_{k}\right|<\epsilon / 2
$$

for $r(\epsilon)<r<1$. Combining this result with (9), we obtain for $r(\epsilon)$ $<r<1$

$$
\left|(1-r) f\left(r e\left(-\alpha_{k}\right)\right)-a_{k}\right|<\epsilon
$$

and since $\epsilon$ is arbitrary, (8) follows immediately.

This result is applicable to the example mentioned in the paragraph before Theorem 1, assuming that for each $\epsilon>0$ the polynomials converge uniformly in a finite union of closed intervals in $[0,1]$ of total length $1-\delta$, with $\delta<\epsilon$. Such will be the case if $g(t)$ is continuous in these closed subintervals (see [2, p. 89]). For then the set $D$ of nonuniform convergence of the approximating polynomials of $g(\nu \alpha)$ is contained in a finite union of intervals of total length $\delta$, and from the uniform distribution of $(\nu \alpha)$ in $[0,1]$ we can conclude that $N_{D} / N \rightarrow \delta$ as $N \rightarrow \infty$. By the same reasoning our results include those of Davenport since his conditions ensure that the Fourier series of $g(t)$ converges uniformly in $\delta \leqq x \leqq 1-\delta$ for each $\delta>0$.

I wish to thank Professor Davenport for his helpful criticisms in the preparation of this note.

\section{REFERENCES}

1. H. Davenport, Note on an irrational power series, Proc. Amer. Math. Soc. 17 (1966), 1-5.

2. A. Zygmund, Trigonometric series, Vol. 1, 2nd ed., Cambridge Univ. Press, New York, 1959.

Mathematisches INSTITUT DER UNIVERSit Ät, GöTtINGEN 